\title{
False-Positive Results in Pregnancy Screening for Aneuploidy
}

\author{
Farnaz Sahhaf ${ }^{1^{*}}$, Arezoo Alimohammadi ${ }^{1}$, Sanaz Mosavi ${ }^{2}$
}

\begin{abstract}
Objectives: The screening of chromosomal aberrations is one of the most common methods during pregnancy although this test is occasionally false-positive.

Materials and Methods: In this case-control study, 252 mothers with false-positive $(n=126)$ and -negative $(n=126)$ tests were evaluated respectively. Demographic data during pregnancy and postpartum included maternal and neonatal complications, and finally, perinatal variables were compared between the two groups.

Results: The mean age of the case group was significantly higher than that of the control group $(P<0.001)$. In addition, lower smoking history in the spouse $(P=0.01)$, higher prevalence of preeclampsia $(P=0.33)$ and proteinuria $(P=0.03)$, preterm infant $(P=0.01)$, and low birth weight $(P=0.04)$ were observed in this group as well. Eventually, the shorter height $P=0.004)$ and lower 5-minute Apgar score $(P=0.03)$ were found in infants.

Conclusions: In general, mothers with positive screening results showed a higher risk of preeclampsia and preterm labor in comparison with those with false-negative screening results.

Keywords: Screening, Down syndrome, Maternal outcomes, Neonatal outcomes
\end{abstract}

\section{Introduction}

Over the past 50 years, prenatal care has developed throughout the world as a clear indication of the promotion of maternal and fetal health.

The cell-free DNA testing has a 60 times higher rate of positive results and lower false-negative tests. These tests could increase diagnostic values by identifying more abnormalities, particularly cases without any diagnostic indications (1). One recent study demonstrated the accuracy of tests in several types of trisomy in twin pregnancies. Interestingly, aneuploidies are most commonly observed in one fetus of twins and thus noninvasive pregnancy tests can be performed as routine prenatal screening tests (2).

The screening of chromosomal diseases is one of the most common methods during pregnancy. Over the past 30 years, methods for detecting and screening chromosomal diseases have changed from invasive to non-invasive techniques (3). Maternal blood variables have a significant effect on the early detection of chromosomal diseases so that these variables in combination with the maternal age and findings of ultrasound during pregnancy result in identifying more than $96 \%$ of genetic disorders and are a good alternative for invasive diagnostic methods such as amniocentesis and cordocentesis (4). These methods are not used as primary diagnostic tools because they are associated with high risks for the mother and fetus.
However, they are administered only for the confirmation of the diagnosis or positive screening tests due to the high risk of genetic diseases (5). Although false-positive results (i.e., positive results without any association with chromosomal abnormalities) are detected, based on previous beliefs, normal pregnancy continues with a negative screening test. Recent evidence has gradually shown that high serum maternal factors can be considered as a risk factor. Various studies have demonstrated that fetal and neonatal mortality rates, preterm labor, low birth weight, and maternal complications increase in such pregnancies (6-9). To the best of our knowledge, no comprehensive study is available regarding evaluating all complications of this type of pregnancy. Therefore, the aim of this study was to investigate maternal, fetal, and neonatal complications of false-positive screening in Down syndrome cases.

\section{Materials and Methods}

This prospectively case-control study was conducted on 210 pregnant women in the first and second trimesters referring to Alzahra teaching hospital for screening during 2016-2017. This study was confirmed by the Ethics Committee of Tabriz University of Medical Sciences. The patients were classified into two groups of $\mathrm{A}$ and $\mathrm{B}$ each containing 126 cases. In the case of the positivity of the screening test, the result was confirmed 


\section{Key Messages}

- Although previous studies evaluated the value of different non-invasive tests in the diagnosis of several types of trisomy in the fetus, fewer studies have focused on maternal and fetal co-morbidities and other outcomes.

- The authors found an insignificant difference between the two groups based on the comparison of the outcomes, therefore, more evaluation is required on larger populations.

by invasive diagnostic tests. Despite positive screening findings, chromosomal abnormalities were not confirmed by genetic tests in terms of amniocentesis, cordocentesis, or chorionic villus sampling. Further, ultrasound findings were evaluated, and embryonic, maternal, and neonatal complications were compared between the two groups. The exclusion criteria were patients without a screening test during the first and second trimesters, twin pregnancies or more, the deficiencies in any parameter in the patient's file, continued care of the pregnancy period, and pregnancy with an uncertain diagnosis.

Different maternal complications were evaluated, including maternal age, weight, history of cigarette smoking, parity, and history of diabetes mellitus or impaired glucose tolerance, positive screenings tests, placenta previa, preeclampsia, and abortion during and after 20th week. Pregnant women were also divided into two groups of preterm (less than 37 weeks) and postterm (more than 37 weeks).

In addition, fetal variables included abortion, fetal mortality, and the number of live births, and the incidence of intrauterine growth retardation (IUGR). Birth height and weight were also recorded based on the weight of newborns in the low birth weight category and small for gestational age. Neonatal infections were studied as well. Then, physical examinations and laboratory tests of neonates, as well as neonatal variables (i.e., neural tube dysfunction, along with eye, gastrointestinal, respiratory, ear and throat, along with genitourinary and musculoskeletal disorders) were evaluated in this study. Data were statistically analyzed by descriptive methods (i.e., frequency, percentage, and mean \pm standard deviation). Finally, Chi-square, Fisher's exact, and MannWhitney $\mathrm{U}$ tests and independent T-test were used to evaluate different findings between the two groups, and a $P$ value less than 0.05 was considered statistically significant.

\section{Results}

This study evaluated 252 cases with false-positive $(n=126)$ and -negative $(n=126)$ screening. Table 1 presents the demographic data of mothers in two groups.

Based on the results, there was a significant relationship
Table 1. Demographic Data of 2 Groups

\begin{tabular}{lccc}
\hline Variables & $\begin{array}{c}\text { False-positive } \\
\text { Screening Test }\end{array}$ & $\begin{array}{c}\text { Negative } \\
\text { Screening Test }\end{array}$ & P Value \\
\hline Age (years) & $33.50 \pm 6.49$ & $28.8 \pm 6.75$ & $<0.001$ \\
Height $(\mathrm{cm})$ & $159.78 \pm 5.81$ & $160.11 \pm 6.95$ & 0.68 \\
Weight $(\mathrm{kg})$ & $75.62 \pm 12.57$ & $76.09 \pm 13.77$ & 0.77 \\
BMI $\left(\mathrm{kg} / \mathrm{m}^{2}\right)$ & $29.64 \pm 4.81$ & $29.72 \pm 5.31$ & 0.90 \\
Gravid & $2.38 \pm 1.34$ & $2.11 \pm 1.27$ & 0.10 \\
Parity & $1.06 \pm 0.09$ & $0.81 \pm 0.08$ & 0.053 \\
Live birth & $1.42 \pm 0.67$ & $1.35 \pm 0.68$ & 0.55 \\
Abortion & $18(14.3 \%)$ & $17(13.5 \%)$ & 0.88 \\
History of smoking & $19(15.1 \%)$ & $35(27.8 \%)$ & 0.01 \\
in spouse & $12(9.5 \%)$ & $10(7.9 \%)$ & 0.65 \\
Hypothyroidism & & &
\end{tabular}

Note. BMI: body mass index.

between age and false-positive screening results although the rate of smoking by the spouse was higher in the control group. Primary screening by double-, triple-, and quadrate-markers in 7 (5.6\%), 2 (1.6\%), and 117 (92.9\%) cases had false-positive results, respectively. In the control group, double-, triple-, and quadrate-marker screening were $26(20.6 \%), 7$ (5.6\%), and 93 (73.8\%) positive cases, respectively.

Table 2 provides pregnancy-induced complications in the two groups. The hematuria was significantly higher in the case group. Although IUGR and fetal death were higher in the case group, they were not significant.

The results further revealed that the neonatal case group had respiratory distress. In the control group, 7 cases suffered from respiratory distress and one case had simultaneously gastrointestinal problems (i.e., imperforated anus, musculoskeletal problems, and foot and mouth disorders). Furthermore, one neonate had a skin disease and the other suffered from cardiovascular disease. The neonatal outcomes between the two groups are shown in Table 3.

\section{Discussion}

Aneuploidy is defined as the structural and numerical aberrations of chromosomes. Among all known recognizable human aneuploidies, trisomy 21 shows the highest frequency of occurrence estimating approximately 1 in 700 live births. The present study evaluated maternal, fetal, and neonatal outcomes to identify if they had fetal positive screening findings. Mothers with a positive screening result were significantly older and their partners' rates of cigarette smoking were lower. Based on the results, preeclampsia, proteinuria, and anterior position of the cord demonstrated significantly false screening results in comparison with the negative screening group. Moreover, IUGR and fetal death were higher in the case group. Fetal positive screening tests were significantly associated with higher delivery of preterm newborns, lower Apgar 
Table 2. The Correlation Between Maternal Complications and the Results of Screening

\begin{tabular}{|c|c|c|c|}
\hline Variables & False-positive Screening Test & Negative Screening Test & $P$ Value \\
\hline Preeclampsia & 27 (21.14\%) & $14(11.3 \%)$ & 0.03 \\
\hline Proteinuria & $33(26.2 \%)$ & $19(15.3 \%)$ & 0.03 \\
\hline \multicolumn{4}{|l|}{ Hypertension } \\
\hline No & $100(79.4 \%)$ & $105(84.7 \%)$ & \multirow{3}{*}{0.14} \\
\hline During pregnancy & $16(12.7 \%)$ & $16(12.9 \%)$ & \\
\hline Chronic & $10(79.4 \%)$ & $3(2.4 \%)$ & \\
\hline \multicolumn{4}{|l|}{ Diabetes mellitus } \\
\hline No & $108(85.7 \%)$ & 111 (91.1\%) & \multirow{3}{*}{0.33} \\
\hline Overt & $12(9.5 \%)$ & $6(4.8 \%)$ & \\
\hline During pregnancy & $6(4.8 \%)$ & $5(4 \%)$ & \\
\hline \multicolumn{4}{|l|}{ Fetal cord position } \\
\hline Anterior & $78(61.9 \%)$ & $48(38.1)$ & \multirow{2}{*}{$<0.001$} \\
\hline Posterior & $48(38.4 \%)$ & $77(61.6)$ & \\
\hline IUGR & $13(10.3 \%)$ & $8(6.3 \%)$ & 0.25 \\
\hline Anomaly confirmed by ultrasound & $8(6.3 \%)$ & $4(3.3 \%)$ & 0.25 \\
\hline Fetal death & $14(11.1 \%)$ & $6(4.76)$ & 0.18 \\
\hline No. of visits during pregnancy & $4.46 \pm 1.49$ & $4.22 \pm 1.72$ & 0.25 \\
\hline \multicolumn{4}{|l|}{ Delivery } \\
\hline Vaginal & $51(40.5 \%)$ & $55(43.7 \%$ & \multirow{2}{*}{0.61} \\
\hline Cesarean section & (59.5\%) & 71 (56.3\%) & \\
\hline
\end{tabular}

Note. IUGR: Intrauterine growth restriction

Table 3. The Correlation Between Screening Results and Newborn Complications in Two Groups

\begin{tabular}{|c|c|c|c|}
\hline Variables & False-positive Screening Test & Negative Screening & $P$ Value \\
\hline \multicolumn{4}{|l|}{ Gender of newborn } \\
\hline Female & $62(49.2 \%)$ & $50.8 \%)) 64$ & \multirow{2}{*}{0.09} \\
\hline Male & $64(50.8 \%)$ & $62(49.2 \%)$ & \\
\hline Preterm & $24(21.4 \%)$ & $3(2.38 \%)$ & 0.01 \\
\hline Fetal death & $5(4.2 \%)$ & $2(1.7 \%)$ & 0.25 \\
\hline The pregnancy age at delivery time & $35.30 \pm 5.74$ & $36.80 \pm 4.46$ & 0.02 \\
\hline Apgar score at $1 \mathrm{~min}$ & $8.37 \pm 1.43$ & $8.66 \pm 1.01$ & 0.07 \\
\hline Apgar score at $5 \mathrm{~min}$ & $9.60 \pm 1.12$ & $9.85 \pm 0.63$ & 0.03 \\
\hline Height (cm) & $47.93 \pm 3.27$ & $49.22 \pm 3.33$ & 0.004 \\
\hline Weight (kg) & $3013.0 \pm 799.58$ & $3119.04 \pm 635.37$ & 0.26 \\
\hline The head circumference $(\mathrm{cm})$ & $33.84 \pm 2.16$ & $34.21 \pm 2.39$ & 0.23 \\
\hline LBW & $14(12.5 \%)$ & $6(5.1 \%)$ & 0.04 \\
\hline SGA & $6(5.4 \%)$ & $5(4.2 \%)$ & 0.69 \\
\hline Macrosomy & $11(9.8 \%)$ & $6(5.1 \%)$ & 0.17 \\
\hline Newborn complications & $12(10.7)$ & $17(14.7 \%)$ & 0.37 \\
\hline
\end{tabular}

Note. LBW: Low birth weight; SGA: Small for gestational age.

scores, and shorter newborns' height. In addition, more fetal deaths, fewer fetal complications, low birth weight and small for gestational age, and the birth of newborns were higher although there was no significant difference between the two groups.

Congenital anomalies and genetic diseases threaten the life of $3 \%$ of newborns by disabilities and deaths (10). According to some studies $(11,12)$, the only way to prevent the delivery of infants with chromosomal abnormalities is the prenatal diagnosis by prenatal screening (i.e., maternal serum markers and ultrasound findings). The higher percentage of the false positive of screening tests was reported in previous studies (6-9). These false-positive results of mothers can be associated with maternal and fetal complications during pregnancy.

Similarly, Pergament et al showed that preeclampsia 
was significantly observed in false-positive screening tests rather than healthy cases (13). Additionally, Dimitrova and Chernev reported that IUGR cases are more common in the false-positive screening group. In addition, abnormal second-trimester screening findings in natural karyotype pregnancies indicated a higher risk of preeclampsia (6). However, Ogle et al (14) and Chapman et al (15) did not suggest statistically significant differences about pregnancy outcomes between individuals with normal and false-positive screening tests. Moreover, Sritippayawan and Vachirasrisoontra (16) found that preeclampsia had no significant difference between the two groups (7.7\% vs. 7.3\%). As shown, previous studies reported inconsistent results about maternal outcomes in terms of fetal positive screenings. However, the risk of maternal complications is generally higher in such situations.

Summers and Huang found that patients with falsepositive screening results had a high incidence of fetal mortality (7). In another study by Dimitrova et al, lower birth weight and early tearing of membranes were more common in false-positive cases. The patients with falsepositive screening results had a higher risk of pregnancy complications $(6,17)$. Based on the findings of Pergament and Stein and Breathnach et al women with false-positive screening results were significantly different from those with screening for preterm delivery and SGA infants $(13,18)$. Chapman et al also demonstrated that falsepositive screening results in women younger than 35 years old were associated with a threefold risk of fetal death (15).

On the contrary, Ogle et al could not observe any difference between the two groups in terms of adverse maternal outcomes (14). Similarly, Pergament et al found no significant difference regarding pregnancy outcomes in comparison with the control group although the incidence of complications in women was less significant in this study (13). However, Sritippayawan and Vachirasrisoontra represented that mothers with falsepositive screening results had a negligible incidence of pregnancy complications (16).

Various studies have contributed to the controversial outcomes of maternal, fetal, and neonatal outcomes of false-positive screening results for Down syndrome cases although contradictory results were reported in this regard. The reason for these differences can be attributed to the sample size of mothers who were evaluated in various studies or the type of screening method. In addition, the reason for the lack of significant differences in some cases may be related to the involvement of other factors such as the age of mothers, the presence of underlying illness, and even the role of a history of abortion in previous pregnancies.

\section{Conclusions}

In general, mothers with false-positive screening results are more prone to preeclampsia and delivery of preterm infants compared to those mothers with negative screening results. However, the incidence of neonatal and fetal death in addition to neonatal complications was not significantly different between the two groups.

\section{Suggestion for Further Research}

According to the results of the current study, more accurate evaluations and appropriate monitoring of mothers with false-positive screening tests are recommended for preventing maternal and fetal complications.

\section{Conflict of Interests}

Authors declare that they have no conflict of interests.

\section{Ethical Issues}

This study was approved by the College of Medicine Institutional Review Board, Tabriz University of Medical Sciences (Ethics No. 57689).

\section{Financial Support}

The authors received no financial support for the research, authorship and publication of this article.

\section{References}

1. Yao H, Gao Y, Zhao J, et al. Genome-wide detection of additional fetal chromosomal abnormalities by cell-free DNA testing of 15,626 consecutive pregnant women. Sci China Life Sci. 2019;62(2):215-224. doi:10.1007/s11427017-9344-7

2. Yu W, Lv Y, Yin S, et al. Screening of fetal chromosomal aneuploidy diseases using noninvasive prenatal testing in twin pregnancies. Expert Rev Mol Diagn. 2019;19(2):189196. doi:10.1080/14737159.2019.1562906

3. Saller DN Jr, Canick JA. Current methods of prenatal screening for Down syndrome and other fetal abnormalities. Clin Obstet Gynecol. 2008;51(1):24-36. doi:10.1097/ GRF.0b013e318160f274

4. Currier R, Wu N, Van Meter K, Goldman S, Lorey F, Flessel $M$. Integrated and first trimester prenatal screening in California: program implementation and patient choice for follow-up services. Prenat Diagn. 2012;32(11):1077-1083. doi:10.1002/pd.3961

5. Barrett SL, Bower C, Hadlow NC. Use of the combined first-trimester screen result and low PAPP-A to predict risk of adverse fetal outcomes. Prenat Diagn. 2008;28(1):28-35. doi:10.1002/pd.1898

6. Dimitrova V, Chernev T, Vragaleva S, et al. [Pregnancy complications with abnormal results of biochemical screening for Down syndrome in second trimester and normal fetal karyotype]. Akush Ginekol (Sofiia). 2002;41(6):3-12.

7. Summers AM, Huang T, Meier C, WyattPR. The implications of a false positive second-trimester serum screen for Down syndrome. Obstet Gynecol. 2003;101(6):1301-1306. doi:10.1016/s0029-7844(03)00235-7

8. Gagnon A, Wilson RD. Obstetrical complications associated with abnormal maternal serum markers analytes. J Obstet Gynaecol Can. 2008;30(10):918-932. doi:10.1016/s1701- 
2163(16)32973-5

9. Jelliffe-Pawlowski LL, Walton-Haynes L, Currier RJ. Using second trimester ultrasound and maternal serum biomarker data to help detect congenital heart defects in pregnancies with positive triple-marker screening results. Am J Med Genet A. 2008;146A(19):2455-2467. doi:10.1002/ ajmg.a.32513

10. Royal College of Obstetricians and Gynaecologists (RCOG). Amniocentesis and Chorionic Villus Sampling. London: RCOG, 2010.

11. Schut W, Weiner P. When to Screen in Obstetrics and Gynecology 1 and 2. Qom: Fanose Andishe Pub; 2011.

12. Cunningham FG, Leveno KJ, Bloom SL, Hauth JC, Rouse DJ, Spong CY. Abortion. In: Williams Obstetrics. New York: McGraw-Hill; 2010.

13. Pergament E, Stein AK, Fiddler M, Cho NH, Kupferminc MJ. Adverse pregnancy outcome after a false-positive screen for Down syndrome using multiple markers. Obstet Gynecol. 1995;86(2):255-258. doi:10.1016/00297844(95)00108-4

14. Ogle R, Jauniaux E, Pahal GS, Dell E, Sheldrake A, Rodeck
C. Serum screening for Down syndrome and adverse pregnancy outcomes: a case-controlled study. Prenat Diagn. 2000;20(2):96-99.

15. Chapman SJ, Brumfield CG, Wenstrom KD, DuBard MB. Pregnancy outcomes following false-positive multiple marker screening tests. Am J Perinatol. 1997;14(8):475-478. doi:10.1055/s-2007-994183

16. Sritippayawan S, Vachirasrisoontra C. Adverse pregnancy outcomes after a false-positive second trimester serum screen for Down syndrome in Thai pregnant women. J Med Assoc Thai. 2005;88(4):449-454.

17. Alamillo CM, Krantz D, Evans M, Fiddler M, Pergament E. Nearly a third of abnormalities found after first-trimester screening are different than expected: 10-year experience from a single center. Prenat Diagn. 2013;33(3):251-256. doi:10.1002/pd.4054

18. Breathnach FM, Malone FD, Lambert-Messerlian G, et al. First- and second-trimester screening: detection of aneuploidies other than Down syndrome. Obstet Gynecol. 2007;110(3):651-657. doi: 10.1097/01. AOG.0000278570.76392.a6

() 2020 The Author(s); This is an open-access article distributed under the terms of the Creative Commons Attribution License (http:// creativecommons.org/licenses/by/4.0), which permits unrestricted use, distribution, and reproduction in any medium, provided the original work is properly cited. 